\title{
Staphylococcus aureus resistente a vancomicina
}

\author{
Carlos Andrés Rodríguez 1,2, Omar Vesga 1,3 \\ 1 Grupo Investigador de Problemas en Enfermedades Infecciosas GRIPE, Medellín, Colombia. \\ 2 Departamento de Farmacología y Toxicología, Facultad de Medicina, Universidad de Antioquia, Medellín, \\ Colombia. \\ 3 Sección de Enfermedades Infecciosas, Departamento de Medicina Interna, Facultad de Medicina, \\ Universidad de Antioquia, Medellín, Colombia.
}

Objetivo. Revisar la evolución y mecanismos moleculares de la resistencia de Staphylococcus aureus a vancomicina.

Fuente de los datos. Se consultó la base de datos MEDLINE y se seleccionaron artículos tipo reportes de caso, estudios bioquímicos, de microscopía electrónica y biología molecular pertinentes.

Síntesis. Después de casi 40 años de eficacia ininterrumpida de la vancomicina, en 1997 se reportaron los primeros casos de fracaso terapéutico debido a cepas de Staphylococcus aureus con resistencia intermedia, denominadas VISA (concentración inhibitoria mínima, CIM, 8 a 16 $\mu \mathrm{g} / \mathrm{ml}$ ), así como a cepas con resistencia heterogénea hVISA (CIM global $=4 \mu \mathrm{g} / \mathrm{ml}$, pero con subpoblaciones VISA), en las cuales la resistencia está mediada por engrosamiento de la pared celular y disminución de su entrecruzamiento, lo que afecta la llegada del antibiótico al blanco principal, los monómeros del peptidoglicano en la membrana plasmática. En 2002 se aisló la primera de las 3 cepas reportadas hasta la fecha con resistencia total al antibiótico, denominadas VRSA $(\mathrm{CIM} \geq 32 \mu \mathrm{g} / \mathrm{ml})$, en las que se encontró el transposón Tn1546 proveniente de Enterococcus spp, responsable del reemplazo de la terminación D-Ala-D-Ala por D-Ala-Dlactato en los precursores de la pared celular con pérdida de la afinidad por el glicopéptido. Conclusiones. La resistencia a vancomicina es una realidad en $S$. aureus, mediada en el caso de VISA por alteraciones en la pared celular que atrapan el antibiótico antes de llegar al sitio de acción, y en el caso de VRSA, por transferencia desde Enterococcus spp. de genes que llevan a la modificación del blanco molecular.

Palabras clave: Staphylococcus aureus, peptidoglicano, biosíntesis, antibióticos glicopéptidos, vancomicina, resistencia microbiana a drogas, resistencia a vancomicina.

\section{Vancomycin-resistant Staphylococcus aureus}

The evolution and molecular mechanisms of vancomycin resistance in Staphylococcus aureus were reviewed. Case reports and research studies on biochemestry, electron microscopy and molecular biology of Staphylococcus aureus were selected from Medline database and summarized in the following review. After almost 40 years of successful treatment of $S$. aureus with vancomycin, several cases of clinical failures have been reported (since 1997). $S$. aureus strains have appeared with intermediate susceptibility (MIC $8-16 \mu \mathrm{g} / \mathrm{ml}$ ), as well as strains with heterogeneous resistance (global MIC $\leq 4 \mu \mathrm{g} / \mathrm{ml}$ ), but with subpopulations of intermediate susceptibility. In these cases, resistance is mediated by cell wall thickening with reduced cross linking. This traps the antibiotic before it reaches its major target, the murein monomers in the cell membrane. In 2002, a total vancomycin resistant strain (MIC $\geq 32 \mu \mathrm{g} / \mathrm{ml}$ ) was reported with vanA genes from Enterococcus spp. These genes induce the change of D-Ala-D-Ala terminus for D-Ala-D-lactate in the cell wall precursors, leading to loss of affinity for glycopeptides. Vancomycin resistance in S. aureus has appeared; it is mediated by cell wall modifications that trap the antibiotic before it reaches its action site. In strains with total resistance, Enterococcus spp. genes have been acquired that lead to modification of the glycopeptide target.

Key words: Staphylococcus aureus; peptidoglycan; biosynthesis; antibiotics, glycopeptide; vancomycin; drug resistance, microbial; vancomycin resistance. 
Staphylococcus aureus es un microorganismo Gram-positivo perteneciente a la familia Micrococcaceae que se distingue de otras especies de estafilococos por la coloración dorada de sus colonias y el resultado positivo en las pruebas de coagulasa, fermentación del manitol y desoxirribonucleasa (1). La descripción clásica de la enfermedad estafilocócica y el papel de la bacteria en la sepsis y formación de abscesos se debe a Sir Alexander Ogston, a finales del siglo XIX (1). En la actualidad $S$. aureus continúa siendo uno de los patógenos más importantes y versátiles de la especie humana, ocupando un lugar preponderante en las infecciones de piel y tejidos blandos, osteomielitis, neumonía, endocarditis y bacteriemias (2), cuyo tratamiento se hace cada vez más complejo debido a la gran capacidad del microorganismo de desarrollar resistencia a los antibióticos (figura 1) mediante una amplia gama de mecanismos que incluyen la producción de enzimas, la síntesis de blancos moleculares de baja afinidad por los antimicrobianos, las mutaciones o modificación enzimática (e.g., metilación) de proteínas o ácidos nucleicos, la generación de formas persistentes de crecimiento lento con defectos en el transporte de electrones (conocidas como variantes de colonias pequeñas) y las alteraciones en la síntesis o la estructura de la pared celular $(2,3)$.

El tratamiento de las infecciones estafilocócicas ha evolucionado desde el inicio de la era antibiótica como consecuencia de la emergencia de la resistencia y el desarrollo de nuevos antibacterianos. La penicilina empezó a utilizarse ampliamente a partir de 1944, y tan solo 2 años después, la resistencia en $S$. aureus mediada por la producción de $\beta$-lactamasas alcanzaba $6 \%$ de los aislamientos; para 1948 este porcentaje superaba $50 \%$ (4). Durante los años 50 se diseminaron las cepas resistentes a la penicilina, así como a los antibióticos recién introducidos eritromicina, cloranfenicol, estreptomicina y

\section{Correspondencia:}

Omar Vesga, Calle 62 \# 52-59 Laboratorio 630. Sede de Investigación Universitaria, Universidad de Antioquia, Medellín, Colombia.

Tel: (4)2106541

ovesgam@medicina.udea.edu.co

Recibido: 14/04/05; aceptado: 26/08/05 tetraciclina (5). El problema se solucionó temporalmente a comienzos de la década del 60 con el desarrollo de las primeras cefalosporinas, cefalotina y cefaloridina, estables frente a la betalactamasa estafilocócica, reemplazadas luego por las 6-acil penicilinas, meticilina, nafcilina y oxacilina. Infortunadamente, en 1961, el mismo año en que la meticilina alcanzó el mercado, se aisló la primera cepa de $S$. aureus resistente (MRSA, por methicillin-resistant S. aureus) (6), productora de una proteína ligadora de penicilina (PBP, por penicillin-binding protein) adicional, $\mathrm{PBP}$ 2A ० 2', con muy baja afinidad por los $\beta$-lactámicos (7). Después de una diseminación inicial durante los años 60, MRSA declinó casi hasta cero en los años 70, para reaparecer a mediados de la década del 80 (8). Desde entonces es un problema creciente en los hospitales, donde alcanza hasta $50 \%$ de los aislamientos (9), y ha empezado a extenderse a la comunidad (10).

Los glicopéptidos han sido el pilar del tratamiento de las infecciones por MRSA desde la introducción de estos antibióticos en 1958; sin embargo, el aislamiento de enterococos resistentes (VRE, vancomycin-resistant Enterococci) y de estafilococos coagulasa-negativos con susceptibilidad disminuida a finales de la década de 1980 (11), hicieron temer la aparición de resistencia en $S$. aureus. Casi una década después, en 1997, se reportó en Japón la primera cepa con una concentración inhibitoria mínima (CIM) para vancomicina de $8 \mu \mathrm{g} / \mathrm{ml}$ (12) y el primer aislamiento con una CIM $>32 \mu \mathrm{g} / \mathrm{ml}$ se registró en Estados Unidos a mediados de 2002 (13).

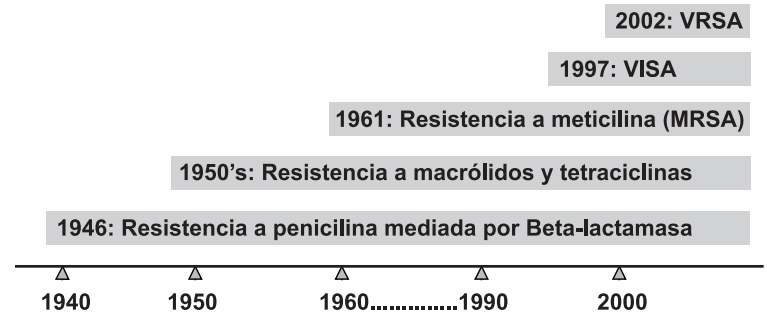

Figura 1. Evolución de la resistencia antimicrobiana en $S$. aureus. Abreviaturas: MRSA: Staphylococcus aureus meticilino-resistente, VISA: Staphylococcus aureus con resistencia intermedia a vancomicina, VRSA: Staphylococcus aureus con resistencia total a vancomicina. 


\section{Definiciones: VISA, hVISA, VRSA}

La literatura sobre la resistencia a vancomicina en $S$. aureus se presta a confusión por las diferencias en los puntos de corte usados en varios países. El CLSI (Clinical Laboratory Standards Institute, antes NCCLS) de los Estados Unidos (14) y la SFM (Société Française de Microbiologie) (15) utilizan 3 categorías: sensible ( $\mathrm{CIM} \leq 4 \mu \mathrm{g} /$ $\mathrm{ml}$ ), intermedio (CIM entre 8 y $16 \mu \mathrm{g} / \mathrm{ml}$ ) y resistente $(\mathrm{CIM} \geq 32 \mu \mathrm{g} / \mathrm{ml})$; mientras que la BSAC (British Society for Antimicrobial Chemotherapy) y el SRGA (Swedish Reference Group for Antibiotics) sólo 2 categorías: sensible (CIM $\leq 4$ $\mu \mathrm{g} / \mathrm{ml})$ y resistente $(\mathrm{CIM} \geq 8 \mu \mathrm{g} / \mathrm{ml})(16,17)$. Esto ha llevado al uso impreciso de los acrónimos VISA (vancomycin-intermediate $S$. aureus) y VRSA (vancomycin-resistant $S$. aureus). Debido a que las cepas VISA y VRSA aisladas son resistentes también a teicoplanina (otro glicopéptido no aprobado en Estados Unidos), se han propuesto los términos GISA y GRSA (por glycopeptideintermediate y glycopeptide-resistant $S$. aureus) como más adecuados; sin embargo, las diferencias entre países en los puntos de corte para este antibiótico, y el aislamiento de cepas resistentes a teicoplanina sensibles a vancomicina, no hacen recomendable el uso de esta terminología. En adelante, la revisión se ceñirá a los acrónimos VISA y VRSA de acuerdo con las definiciones del CLSI (14).

Existe otra categoría correspondiente a cepas de $S$. aureus con $\mathrm{CIM} \leq 4 \mu \mathrm{g} / \mathrm{ml}$ (sensibles) en las pruebas estándar, pero que presentan subpoblaciones con CIM 8-16 $\mu \mathrm{g} / \mathrm{ml}$ al realizar un perfil de análisis poblacional (PAP, del inglés population analysis profile). Estas cepas se han denominado hetero-VISA (hVISA) (ver más adelante).

\section{Resistencia intermedia a vancomicina: cepas VISA}

En 1997, Hiramatsu et al. (12) reportaron el aislamiento en Japón de una cepa MRSA en un paciente de 4 meses de edad sometido a cirugía cardiaca quien, después del procedimiento, presentó fiebre y signos de infección en la herida quirúrgica. Recibió 29 días de terapia con vancomicina ( $45 \mathrm{mg} / \mathrm{kg} /$ día) sin respuesta, por lo que se agregó arbekacina (aminoglicósido aprobado en Japón para MRSA). Después de una mejoría inicial reapareció la fiebre y se formó un absceso subcutáneo, por lo que el tratamiento se cambió a ampicilina-sulbactam más arbekacina, y el paciente fue dado de alta 17 días después. La cepa MRSA aislada, denominada Mu50, tenía una CIM para vancomicina de $8 \mu \mathrm{g} / \mathrm{ml}$ determinada por microdilución en caldo. Desde entonces se han reportado cepas VISA en Europa $(18,19)$, Estados Unidos (20-23), Asia (24) y Brasil (25). El grupo colombiano de resistencia antimicrobiana, RESCOL (26), tras analizar 296 aislamientos nosocomiales de S.aureus en el período 2001-2002 encontró 52\% de resistencia a meticilina, pero ninguna cepa VISA mediante la técnica de tamizaje en agar infusión cerebro corazón (BHIA, Brain-Heart infusion Agar) con 6 $\mu \mathrm{g} / \mathrm{ml}$ de vancomicina según recomendación del CLSI y de los Centers for Disease Control (CDC) (27). Debe anotarse que el método empleado por los investigadores de RESCOL para detectar VISA tiene una muy baja sensibilidad $(22 \%$, ver adelante).

Los casos reportados de infecciones por VISA no permiten establecer factores de riesgo específicos; sin embargo, se encuentran algunos puntos comunes: los pacientes presentaban enfermedades de base similares (cáncer, diabetes mellitus e insuficiencia renal), la mayoría habían sido sometidos a diálisis y tenían bacteriemias asociadas con catéteres o material protésico, y habían sido expuestos a dosis plenas de vancomicina por períodos muy prolongados (entre 6 y 18 semanas) 3 a 6 meses antes de la detección de la infección por VISA (28). Además, los reportes de los Estados Unidos sugieren que las cepas VISA se desarrollaron a partir de cepas de MRSA que infectaron previamente los pacientes, dada la identidad entre sus patrones de electroforesis de campo pulsado (PFGE, pulsedfield gel electrophoresis) (21-23).

La vancomicina es un antibiótico del grupo de los glicopéptidos que tiene como blanco principal las subunidades D-Ala-D-Ala en los monómeros del peptidoglicano de las bacterias Gram positivas, que sirven como precursores de la síntesis de la pared celular. La resistencia en las cepas VISA 
no está mediada por un mecanismo único, sino que parece involucrar una compleja reorganización de la síntesis de la pared celular. A continuación revisaremos el mecanismo de síntesis de la pared celular de $S$. aureus (figuras 2 y 3 ), y los ajustes que hace la bacteria a su metabolismo antes de desplegar el fenotipo resistente.

El principal componente de la pared celular de $S$. aureus es el peptidoglicano, una matriz de polisacárido compuesta de subunidades alternantes de $\mathrm{N}$-acetilglucosamina (GlcNAc) y ácido $\mathrm{N}$-acetilmurámico (MurNac). El paso inicial (figura 2) en la producción del monómero de peptidoglicano es la formación de glucosamina6-fosfato $\left(\mathrm{GlcNH}_{2}-6-\mathrm{P}\right)$ a partir de glucosa, fructosa o glucosamina importadas al citoplasma desde el medio exterior. Luego $\mathrm{GlcNH}_{2}-6-\mathrm{P}$ se modifica en $\mathrm{GlcNH}_{2}-1-\mathrm{P}$ y es acetilada para formar UDP-GlcNAc, la cual se combina con enolpiruvato derivado de glucosa para formar UDP-MurNAc. Una L-alanina se incorpora luego al residuo UDPMurNac, seguida por D-glutamato, L-lisina, y Dalanil-D-alanina para formar un pentapéptido, constituyendo así el nucleótido de Parks (29). Este es luego transferido al $\mathrm{C}_{55}$ undecaprenilfosfato para formar el lípido I del transportador de membrana del peptidoglicano, liberando UMP en el proceso (30). El siguiente paso implica la transferencia secuencial de 5 residuos de glicina al componente L-lisina del pentapéptido para formar una cadena lateral de pentaglicinas. El residuo D-glutamato es luego transformado por amidación en Dglutamina, usando L-glutamina como donador de $\mathrm{NH}_{4}+$, reacción que sólo ocurre si la concentración de este aminoácido en la célula es alta (31). A continuación se agrega GlcNAc al complejo $\mathrm{C}_{55}$ undecaprenil-P-P-MurNAc usando UDPGlcNAc como donante, formándose el lípido II, que es transferido a la cara externa de la membrana citoplasmática, donde las transglicosilasas unen el precursor GlcNAc-MurNAc-pentapéptidopentaglicina a las cadenas existentes de peptidoglicano (figura 3 ). Más tarde, las transpeptidasas unen la cadena de pentaglicina de un monómero al residuo D-alanina en la penúltima posición del pentapéptido de la cadena vecina, liberándose el último residuo de $\mathrm{D}$-alanina y dando origen a un entrecruzamiento de cadenas que confiere rigidez a la estructura (30). El paso final en el procesamiento de la pared celular es realizado por carboxipeptidasas, que remueven

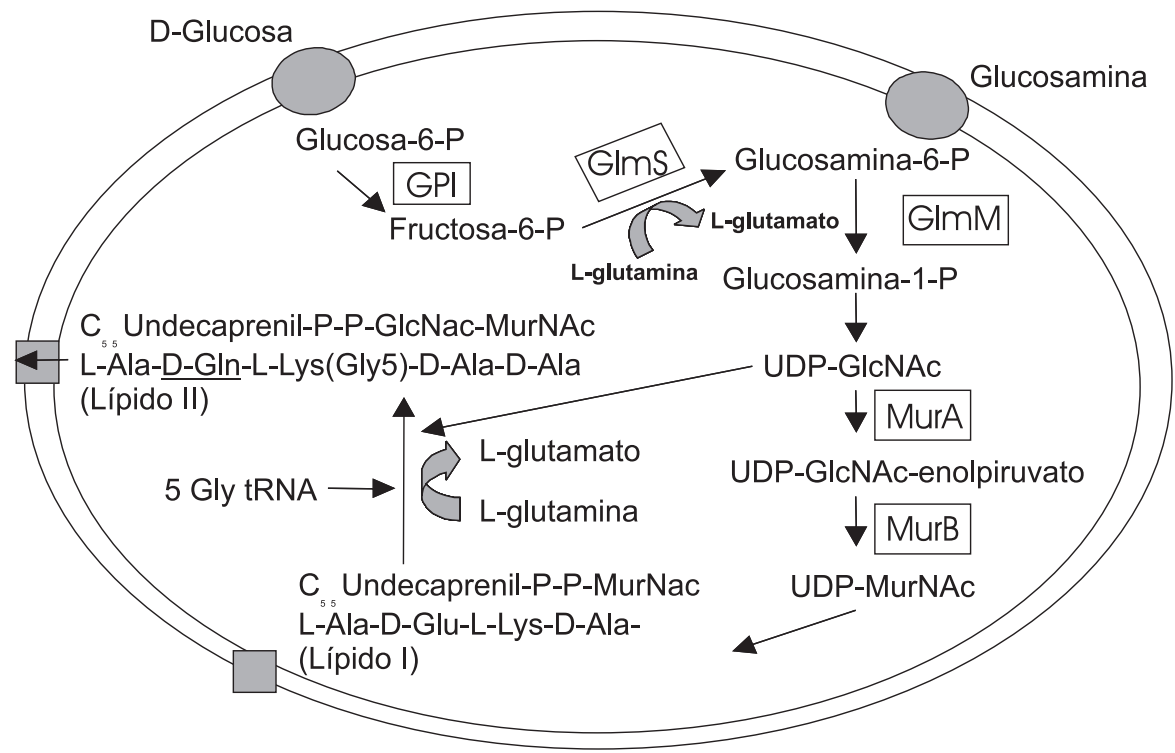

Figura 2. Producción de los monómeros de peptidoglicano en $S$. aureus. Abreviaturas, GPI: glucosa-6-P isomerasa, GlmS: glucosamina sintetasa, GlmM: glucosamina-6-P mutasa, MurA: UPD-GlcNAc-enolpiruvil transferasa, MurB: UDP-MurNAcdeshidrogenasa. (Modificada de Avison et al. (29) con permiso de Oxford University Press). 

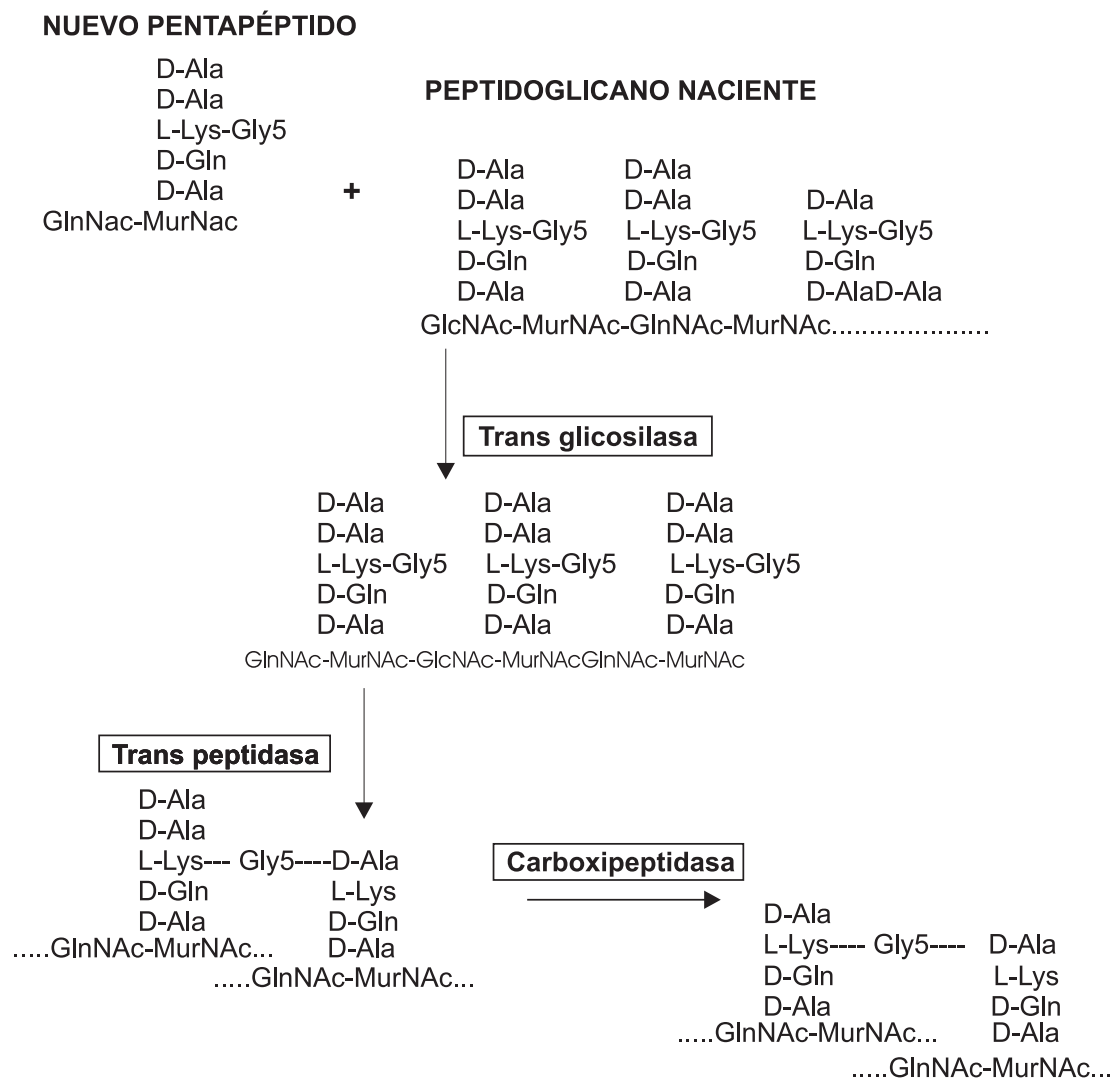

Figura 3. Procesamiento del peptidoglicano en S. aureus. Las subunidades nuevas de GlcNAc-MurNAc-pentapéptidopentaglicina son unidas por las transglicosilasas y luego incorporadas a la red existente de peptidoglicano por medio de las transpeptidasas que entrecruzan los pentapéptidos con las pentaglicinas. El paso final de las carboxipeptidasas es necesario solamente si el pentapéptido terminal no es entrecruzado. La presencia de D-glutamina (D-Gln en lugar de D-Glu) es necesaria para una transpeptidación eficiente. (Modificada de Avison et al. (29), con permiso de Oxford University Press).

las alaninas terminales en las unidades que no fueron entrecruzadas por las transpeptidasas (30). Como no todos los pentapéptidos son utilizados, siempre queda un número de residuos D-Ala-DAla sin procesar en la pared celular que se estima llegan a ser $6 \times 10^{6}$ por cada célula de $S$. aureus (31).

Existen por tanto 2 tipos de blanco para la vancomicina: en primer lugar, los residuos libres de D-Ala-D-Ala en las capas terminadas de peptidoglicano y, en segundo, los monómeros de peptidoglicano que emergen de la membrana plasmática, los cuales son el sustrato de las transglicosilasas (32). La unión del glicopéptido a los primeros blancos no interfiere con la síntesis de peptidoglicano, pero sí con la transpeptidación mediada por las PBP; en cambio, si el antibiótico se une a los monómeros en la membrana, la síntesis de la pared celular se detiene y las células dejan de multiplicarse. Es muy importante anotar que para llegar a estos monómeros, las moléculas de vancomicina tienen que atravesar por lo menos 20 capas de peptidoglicano sin ser atrapadas por los primeros blancos (33).

El mecanismo de resistencia a vancomicina ha sido estudiado extensamente en la primera cepa VISA, Mu50 $(33,34)$ (cuadro1). Las pruebas bioquímicas y la microscopía electrónica indican que esta cepa produce mayores cantidades de peptidoglicano (debido a la mayor incorporación de GlcNAc, la mayor concentración de monómeros de peptidoglicano y la mayor producción de PBP2 
Cuadro 1. Mecanismos implicados en la resistencia intermedia a vancomicina.

\begin{tabular}{lc}
\hline Mecanismos & Referencias \\
\hline Engrosamiento de la pared celular & $33,35,36$ \\
- Aumento en la captación de GlcNAc & \\
- Incremento de monómeros de peptidoglicano \\
- Sobreexpresión de PBP 2 y $2 a$ \\
$\begin{array}{l}\text { Disminución del entrecruzamiento } \\
\text { - Disminución en la amidación de los } \\
\text { muropéptidos } \\
\text { - Reducción en la expresión de PBP4 }\end{array} 34,35,41,43$
\end{tabular}

y $2 A)$, y que su pared celular es más gruesa (entre 30 y 40 capas de peptidoglicano). Como consecuencia, un mayor número de moléculas de vancomicina pueden ser atrapadas antes de llegar a la membrana citoplasmática, donde actúan las transglicosilasas. Este mecanismo se ha denominado "atrapamiento de afinidad". Adicionalmente, se ha visto que la estructura externa de la pared celular se distorsiona por las moléculas secuestradas de vancomicina, lo que impide aun más la entrada de otras moléculas del antibiótico (35). Este hallazgo fundamental no se limita a la cepa Mu50. Cui et al. (36) midieron por microscopía electrónica el grosor de la pared celular de 16 cepas VISA recuperadas en siete países diferentes, obteniendo una media de 31,3 nm (desviación estándar 2,62) y las compararon con 7 cepas susceptibles VSSA (vancomycinsusceptible $S$. aureus) cuyo grosor medio fue de $23,99 \mathrm{~nm}$ (desviación estándar 2,04). Además, encontraron una correlación significativa $(r=$ $0,908, p<0,001$ ) entre la CIM para vancomicina y el grosor de la pared.

Además del engrosamiento de la pared, se ha observado una disminución en el grado de entrecruzamiento (cross-linking) de las cadenas de peptidoglicano (cuadro 1), lo cual aumenta el número de D-Ala-D-Ala libres en las capas externas de la pared y por tanto más antibiótico puede ser atrapado antes de llegar al sitio de acción (31). Se han planteado 2 hipótesis para explicar este fenómeno: la disminución de la amidación de los muropéptidos y la reducción en la expresión de la PBP4.

Como ya se mencionó, durante las fases previas a la transferencia de los precursores de la pared celular a través de la membrana, el D-glutamato del pentapéptido es convertido por amidación (a partir de L-glutamina que se transforma en Lglutamato al donar el grupo $\mathrm{NH}_{4}+$ ) en D-glutamina. Sin embargo, en Mu50 se encuentra una mayor proporción de muropéptidos no amidados (34), los cuales se consideran pobres sustratos para las transpeptidasas, lo que, en consecuencia, genera menos entrecruzamientos (35). El anabolismo del peptidoglicano es altamente dependiente de la disponibilidad de L-glutamina, que actúa como donante de grupos $\mathrm{NH}_{4}+$ para convertir fructosa6-fosfato en $\mathrm{GlcNH}_{2}-6$-P en la producción de UDPGlcNAc, y para producir UDP-MurNAc por medio del fosfoenolpiruvato (figura 2). La regeneración de L-glutamina a partir de L-glutamato está mediada por la enzima glutamina sintetasa, que es una de las más estrictamente reguladas en las bacterias (37). La vía de regulación de la glutamina sintetasa en $S$. aureus no se conoce, pero las vías intermedias son las mismas que en Escherichia coli, en la cual la enzima es inhibida por la $\mathrm{GlcNH}_{2}-6-\mathrm{P}$ y activada por la presencia de 2 -cetoglutarato o L-glutamato $(37,38)$. En la cepa Mu50 existe evidencia de que la célula utiliza preferencialmente glucosa en lugar de glucosamina para producir peptidoglicano $y$, por tanto, las reservas de L-glutamina se depletan debido a la mayor producción de $\mathrm{GlcNH}_{2}-6-\mathrm{P}$ y, subsecuentemente, GlcNAc (35). Lo anterior lleva a que la $L$-glutamina disponible para la amidación del D-glutamato en el pentapéptido de los precursores también disminuya y, en consecuencia, haya una menor transpeptidación (39).

Con respecto a la expresión de PBP4, los experimentos de Sieradzki y Tomasz $(40,41)$ resultaron en la obtención por subcultivo secuencial de 2 mutantes altamente resistentes a vancomicina (CIM $72 \mu \mathrm{g} / \mathrm{ml})$, denominadas VM y TNM. La resistencia se relacionaba con el engrosamiento de la pared celular y la liberación al medio de grandes cantidades de peptidoglicano con una disminución hasta de $85 \%$ en su grado de entrecruzamiento $y$, por tanto, una mayor cantidad de D-Ala-D-Ala libre para atrapar el antibiótico. En ambas cepas la expresión de PBP4, una carboxipeptidasa y transpeptidasa secundaria que se requiere para el 
entrecruzamiento de la pared celular (42), estaba disminuida o anulada. Se observaron resultados similares en cepas clínicas de VISA en estudios de Finan et al. (43). Aunque las hipótesis mencionadas explican parcialmente el mecanismo de resistencia a vancomicina, se han aislado unas pocas cepas VISA que no tienen estas alteraciones en la pared celular, lo que hace pensar que la resistencia debe obedecer a mecanismos alternativos aún sin estudiar (44).

\section{Estudios genómicos en S. aureus Mu50}

La activación de la síntesis de la pared celular observada en la cepa Mu50 debe ocurrir en múltiples puntos de la vía metabólica, por lo cual la mutación de un sólo gen o la adquisición de un gen exógeno es una explicación poco probable. En su lugar, la sobreexpresión de varios genes asociados a la síntesis del peptidoglicano sería una hipótesis más factible. Kuroda et al. (45) prepararon librerías de ADNc de la cepa Mu50 y de una cepa sensible a vancomicina, y hallaron varios genes sobreexpresados. En primer lugar, se identificó un gen denominado vraR (por vancomycin resistance associated), similar al gen yvqC de Bacillus subtilis, conocido como un regulador de respuesta, que al introducirse mediante un vector a la cepa control elevó su CIM de 0,5 a 2,0 $\mu \mathrm{g} /$ $\mathrm{ml}$. También encontraron 3 genes con alta similitud con el operón de fructosa en $B$. subtilis (fruR, fruB, fruA), 2 genes involucrados en la síntesis de ácidos grasos $(v r a A-C)$ y 4 genes de nuevos transportadores de la familia ABC: ATP-binding cassettes (vraDE, vraFG), muy similares al transportador de glutamina glnQ. Estos hallazgos pueden relacionarse con el direccionamiento del metabolismo de los azúcares glucosa y fructosa hacia la síntesis de la pared celular, y con una mayor captación de nutrientes del medio para mantener su alta tasa de producción.

Otra aproximación presentan Avison et al. (29) basándose en la comparación de las secuencias publicadas del genoma completo de Mu50 y de varias cepas MRSA sensibles a vancomicina (46). El análisis in silico reveló 17 mutaciones de pérdida de función en genes con funciones ya caracterizadas. Se destaca la completa disrupción del gen murA, que codifica la UDP-GIcNAc- enolpiruvil transferasa, necesaria para la síntesis del UDP-MurNAc (47); del gen mrp, análogo de $f m t B$, gen cuya inactivación disminuye la resistencia a meticilina en cepas MRSA (48), y de los genes odhA y $s d h B$, que codifican por las enzimas 2-cetoglutarato deshidrogenasa y succinato deshidrogenasa, respectivamente. Es notable el hallazgo de estas deleciones en enzimas encargadas de reacciones clave en la síntesis de la pared celular y de metabolitos intermediarios de la glutamina y la glucosamina, lo que está en concordancia con las alteraciones bioquímicas reportadas en la cepa Mu50 (33). También es destacable la pérdida del gen mutS, que codifica una proteína reparadora del ADN. Estudios anteriores han mostrado que la pérdida de la función de este gen conduce a un fenotipo de hipermutación (100 veces más mutaciones espontáneas comparadas con las cepas mutS+), asociado al desarrollo de resistencia a antibióticos y a una disminución de la adaptabilidad evolutiva (fitness, en la literatura inglesa) (49), lo cual puede explicar los mayores tiempos de generación reportados para la cepa Mu50 en comparación con cepas VSSA (36).

\section{Resistencia intermedia heterogénea a vancomicina: cepas hVISA}

El fenómeno de la heterorresistencia, o sea, la distribución no homogénea del fenotipo resistente en una población bacteriana, se conoce en $S$. aureus desde el desarrollo de la resistencia a meticilina (50). A comienzos de los años 1980 se observó que si bien todas las células de MRSA en una población dada poseían el gen mecA, la CIM de meticilina variaba desde unos pocos microgramos por mililitro por encima del punto de corte de susceptibilidad hasta niveles cientos de veces más altos, hecho que se atribuye a la expresión diferencial de otros determinantes genéticos involucrados en la resistencia, tales como el gen femA (51). Se postula que el uso de carbapenems y cefalosporinas de tercera generación para tratar estas cepas MRSA heterogéneas, que al principio aparecían susceptibles, condujo a la selección de las subpoblaciones más resistentes y al predominio de MRSA homogéneo (52). En el caso de vancomicina, las cepas hVISA tienen 
subpoblaciones de células con resistencia intermedia (CIM entre 8 y $16 \mu \mathrm{g} / \mathrm{ml}$ ), mientras la CIM global está en el rango de susceptibilidad $(\leq 4 \mu \mathrm{g} / \mathrm{ml})$. La vancomicina crea una presión selectiva que favorece el predominio de las subpoblaciones de células más resistentes, generando hVISA y, eventualmente, con la exposición continua, una población uniforme de VISA (53). La primera cepa hVISA, Mu3, fue aislada en Japón en 1996 en un paciente de 64 años con neumonía por MRSA tratada sin éxito con vancomicina durante 12 días, con agravamiento del cuadro en los últimos 4 días de terapia. Aunque la CIM de este aislamiento era de $4 \mu \mathrm{g} / \mathrm{ml}$, algunas subpoblaciones podían crecer en medios con vancomicina a concentraciones entre 5 y $9 \mu \mathrm{g} / \mathrm{ml}$, indicando la presencia de resistencia heterogénea. La electroforesis de campo pulsado mostró que esta cepa tenía un patrón indistinguible de Mu50, aislada unos meses después en el mismo hospital, lo que sugiere que Mu3 puede ser su origen. Además, el cultivo seriado de la cepa hVISA en concentraciones crecientes de vancomicina dio origen a subpoblaciones con un nivel de resistencia similar al de Mu50. Este fenómeno in vitro es sugestivo de que la colonización o infección con VISA puede ser precedida por infección con hVISA, y luego la exposición prolongada al antibiótico lleva a la aparición de una población con resistencia uniforme (54).

Actualmente no hay un método estandarizado para identificar las cepas hVISA. El análisis poblacional se ha propuesto como la técnica más precisa. En éste, las diluciones seriadas de una suspensión inicial de $10^{8} \mathrm{UFC} / \mathrm{ml}$ se siembran en cajas de Petri con BHIA con vancomicina (BHIA-V) a concentraciones crecientes y a las 48 horas se determina el número de colonias viables, registrando los datos en una gráfica semilogarítmica. Sin embargo, la naturaleza laboriosa de esta técnica y el requerimiento de un costoso equipo electrónico para siembra espiral en medio sólido (Spiral Plating Apparatus), la hacen poco práctica para la mayoría de laboratorios. En su lugar, Hiramatsu et al. (54) propusieron una técnica simplificada que consiste en inocular $10 \mu \mathrm{l}$ de una suspensión de $10^{8} \mathrm{UFC} / \mathrm{ml}$ en BHIA con $4 \mu \mathrm{g}$ de vancomicina por $\mathrm{ml}$ (técnica BHIA-V4). El crecimiento a las 24 horas se considera VISA potencial y a las 48 horas hVISA potencial. El estado hVISA se confirma si se encuentran subpoblaciones con CIM de $8 \mu \mathrm{g} / \mathrm{ml}$ que permanecen estables durante más de 9 días en medio libre de antibiótico. Otros métodos reportados en la literatura utilizan medios diferentes como el agar Mueller-Hinton (MHA), o varían los inóculos y las concentraciones de vancomicina. Walsh et al. (55) probaron 284 cepas de MRSA y 45 cepas de VISA y hVISA comparando las técnicas de BHIA-V4, MHA con $5 \mu \mathrm{g} / \mathrm{ml}$ de vancomicina, dilución en caldo, dilución en agar, y el E-test, con el PAP como estándar de oro. La sensibilidad y especificidad encontradas fueron: BHIA, 22 y 97\%; MHA, 20 y $99 \%$, dilución en caldo, 11 y $100 \%$, y dilución en agar, 20 y $100 \%$, mientras que el E-test con un inóculo de 2,0 McFarland alcanzó $96 \%$ y $97 \%$, respectivamente.

Los reportes de hVISA son más frecuentes entre cepas resistentes a la meticilina. De 7.920 cepas de $S$. aureus aisladas en diferentes países en el período de 1997 a 2001, 1,67\% (132 aislamientos) fue reportado como hVISA (53), aunque esta frecuencia puede ser mayor dada la carencia de un método estandarizado y sensible para la detección de heterorresistencia. En el mismo estudio se encontró una frecuencia 43 veces mayor de hVISA en las cepas meticilino resistentes (131 entre $6.052,2,16 \%$ ) que en las cepas meticilino sensibles de $S$. aureus (1 entre $1.868,0,05 \%$ ), hecho sugestivo de que estas cepas más expuestas a los antibióticos tendrían mayor capacidad para desarrollar resistencia heterogénea.

La significancia clínica de hVISA no se ha definido con exactitud, en particular debido a que algunos consideran que la vancomicina tiene menor efecto bactericida contra $S$. aureus que los $\beta$-lactámicos y que su penetración al sitio de la infección es limitada por el gran tamaño de la molécula, características que podrían por sí solas explicar las fallas terapéuticas observadas en infecciones por estas cepas $(56,57)$. Desde un punto de vista biológico, el status hVISA conferiría una ventaja de supervivencia a $S$. aureus, ya que si bien dosis 
terapéuticas de vancomicina eliminan 99,9\% de la población, la fracción restante sobrevive y es capaz de crecer a concentraciones de antibiótico de $4 \mu \mathrm{g} / \mathrm{ml}$ o superiores. Estas células VISA gastan gran cantidad de energía para engrosar la pared celular y tienen una adaptabilidad evolutiva (fitness) menor que las cepas VSSA, por lo cual tienden a revertir al fenotipo hVISA una vez cesa la presión del antibiótico. Sin embargo, cuando se exponen nuevamente a vancomicina generan mutantes resistentes con una alta frecuencia, lo que asegura la supervivencia de la cepa (58).

\section{Resistencia total a vancomicina: cepas VRSA}

En 1992, Noble et al. (59) reportaron la transferencia in vitro y sobre la piel de un ratón de los genes de resistencia a vancomicina de una cepa de Enterococcus faecalis a $S$. aureus, confiriéndole resistencia total $(\mathrm{CIM} \geq 32 \mu \mathrm{g} / \mathrm{ml})$. Desde entonces se ha postulado que puede ocurrir transferencia de material genético si los dos microorganismos comparten el mismo nicho ecológico. Al respecto, estudios de Ray et al. (60) demostraron que hasta $62 \%$ de los pacientes colonizados con VRE tienen también $S$. aureus en el tracto gastrointestinal, constituyendo un reservorio potencial para la aparición de cepas resistentes.

En junio de 2002 se aisló la primera cepa clínica de VRSA (13), con una CIM para vancomicina de $1.024 \mu \mathrm{g} / \mathrm{ml}$, en una paciente de 40 años residente en Detroit, Michigan, Estados Unidos, con diagnóstico de hipertensión, diabetes mellitus, enfermedad vascular periférica y falla renal crónica en hemodiálisis, quien había recibido vancomicina previamente para tratar infecciones recurrentes de úlceras en miembros inferiores por MRSA y $E$. faecalis. Después de una amputación, la paciente desarrolló bacteriemia por MRSA y un absceso asociado a un injerto arteriovenoso para acceso de diálisis. El injerto fue retirado y la diálisis se realizó por medio de catéteres temporales colocados secuencialmente. El sitio de acceso del tercer catéter mostró signos de infección, por lo que fue retirado y de la punta se aislaron $S$. aureus y $E$. faecalis resistentes a vancomicina. Los mismos microorganismos se aislaron en 2 úlceras plantares. Después de desbridamiento quirúrgico y 14 días de trimetoprim-sulfametoxazol más metronidazol, la paciente fue dada de alta y, para diciembre de 2002, las úlceras habían sanado. Los cultivos de las axilas, narinas, ombligo y recto fueron negativos para VRSA y no se aisló el microorganismo en ninguno de los contactos del paciente (61). Dos meses después se reportó la segunda cepa VRSA (62) en un paciente de 70 años residente en Hershey, Pennsylvania, quien padecía obesidad mórbida y una úlcera crónica en el tobillo, en la cual se había aislado previamente MRSA y VRE. Sorprendentemente, el paciente no había sido hospitalizado ni había recibido vancomicina en los 5 años previos (63). El tercer caso de VRSA se reportó en marzo de 2004 en un paciente hospitalizado en Nueva York $(\mathrm{CIM}>256 \mu \mathrm{g} / \mathrm{ml})$, que se encuentra actualmente en estudio por parte de las autoridades sanitarias (64).

La característica común de los 3 aislamientos es la presencia del fenotipo VanA, que confiere resistencia a vancomicina mediante la sustitución del extremo D-Ala-D-Ala del monómero de peptidoglicano por D-Ala-D-Lactato, cuya afinidad por el antibiótico es 1.000 veces menor que la del monómero silvestre. La resistencia está mediada por un complejo de genes: vanS, vanR (genes reguladores), vanA, vanH, vanX (genes efectores). La proteína VanS se autofosforila en presencia de vancomicina y transfiere el grupo fosfato a VanR, que es un factor de transcripción para los demás genes. VanX es una dipeptidasa que hidroliza los residuos D-Ala-D-Ala para que no se incorporen a la pared celular blancos susceptibles a vancomicina. VanA cataliza la formación de DAla-D-Lactato y $\mathrm{VanH}$, una alfa-cetoácido reductasa que se encarga de aportar el D-lactato necesario (65). El análisis molecular del primer aislamiento reveló la presencia de un plásmido conjugativo en el cual estaba integrado el gen $\operatorname{vanA}(66)$, con $100 \%$ de homología con la secuencia prototipo aislada en VRE, el transposón Tn1546, demostrándose así la transferencia interespecies de este determinante de resistencia, que además es transferible a otras cepas de $S$. aureus. El análisis del segundo aislamiento (67) mostró igualmente la presencia del transposón Tn1546, pero con algunas secuencias truncadas, 
lo que podría explicar el menor nivel de resistencia de esta cepa (CIM $32 \mu \mathrm{g} / \mathrm{ml} v \mathrm{~s}$. $1024 \mu \mathrm{g} / \mathrm{ml}$ del primer aislamiento).

\section{Conclusiones}

Las cepas VISA y hVISA han desarrollado un mecanismo de resistencia completamente nuevo, consistente en el atrapamiento de la vancomicina en la pared celular bacteriana, por el cual el antibiótico retiene su estructura y actividad biológica, pero pierde la disponibilidad para interactuar con el blanco molecular. Adicionalmente, las observaciones sobre el carácter reversible del fenotipo resistente y la disminución de fitness que acarrea la resistencia han llevado a autores como Hiramatsu (58) a postular la dinámica hVISA-VISA como una estrategia evolutiva que le permite a $S$. aureus adaptarse en ambientes con alta presión de antibióticos (en este caso glicopéptidos), manteniendo un equilibrio entre la supervivencia de la cepa y el alto costo biológico de la resistencia. Esta disminución de la adaptabilidad evolutiva de las cepas VISA es probablemente la razón por la cual no se han diseminado, pero la acumulación de mutaciones adicionales que compensen tal desventaja evolutiva es una posibilidad latente, como se ha evidenciado en el caso de E. coli y Mycobacterium tuberculosis resistentes a estreptomicina, las cuales, después de sufrir un déficit inicial en la velocidad de crecimiento, llegan a igualar o incluso superar a sus contrapartes sensibles tras un periodo de replicación en medio libre de antibiótico (68). De otro lado, la presión continua de los antibióticos y la coexistencia de microorganismos que con frecuencia creciente portan mecanismos de resistencia transferibles y más eficientes (Enterococcus spp.) han hecho inevitable la aparición de las cepas VRSA, escasas hasta la fecha, pero con un peligroso potencial de diseminación.

Finalmente, aunque la resistencia a vancomicina en $S$. aureus es una realidad, la situación hasta ahora no es tan crítica como se pronosticaba (69), ya que las cepas encontradas no son panresistentes $y$, de hecho, han sido tratadas exitosamente con antibióticos de amplio uso clínico como trimetoprim-sulfametoxazol, o recién aprobados, como linezolid. Además, estas cepas son susceptibles a casi todos los antibióticos desarrollados recientemente contra cocos Grampositivos, incluidos quinupristina/dalfopristina, daptomicina, oritavancina y tigeciclina. Sin embargo, es de suma importancia implementar estrategias rigurosas de monitorización para detectar y tratar rápidamente nuevos aislamientos, aplicar medidas adecuadas de barrera para evitar la diseminación, e insistir incansablemente en el uso prudente de los antimicrobianos (70).

\section{Agradecimientos}

Agradecemos a los estudiantes del Grupo Investigador de Problemas en Enfermedades Infecciosas, GRIPE, por su colaboración durante la búsqueda bibliográfica.

\section{Conflictos de interés}

Los autores son profesores (asistente y asociado respectivamente) de la Facultad de Medicina de la Universidad de Antioquia y declaran que no se presentaron conflictos de interés en la elaboración de este manuscrito.

\section{Financiación}

Aparte de su asignación como docentes de la Facultad de Medicina de la Universidad de Antioquia, los autores no recibieron ninguna financiación para la realización de este artículo.

\section{Referencias}

1. Lowy FD. Staphylococcus aureus infections. N Engl J Med 1998;339:520-32.

2. Waldvogel FA. Staphylococcus aureus (including staphylococcal toxic shock). In: Mandell GL, Bennett JE, Dolin R, editors. Principles and practice of infectious diseases. Fifth Edition. Philadelphia: ChurchillLivingston; 2000. p.2069-92.

3. Vesga O, Groeschel MC, Otten MF, Brar DW, Vann JM, Proctor RA. Staphylococcus aureus small colony variants are induced by the endothelial cell intracellular milieu. J Infect Dis 1996;173:739-42.

4. Barber M, Rozwadowska-Dowzenko M. Infection by penicillin-resistant staphylococci. Lancet 1948;252:641-4.

5. Williams RE. Epidemic staphylococci. Lancet 1959;273:190-5.

6. Jevons MP. Calbenin-resistant staphylococci. Br Med J 1961;1:124-5.

7. Hartman BJ, Tomasz A. Low affinity penicillin-binding protein associated with beta-lactam resistance in Staphylococcus aureus. J Bacteriol 1984;158:513-6. 
8. Ayliffe GA. The progressive intercontinental spread of methicillin-resistant Staphylococcus aureus. Clin Infect Dis 1997;24:S74-9.

9. Diekema DJ, Pfaller MA, Schmitz FJ, Smayevsky J, Bell J, Jones RN et al. Survey of infections due to Staphylococcus species: frequency of occurrence and antimicrobial susceptibility of isolates collected in the United States, Canada, Latin America, Europe and the Western Pacific region for the SENTRY antimicrobial surveillance program 1997-1999. Clin infect Dis 2001;32:114-32.

10. Centers for Disease Control and Prevention. Four pediatric deaths from community-acquired methicillinresistant Staphylococcus aureus. JAMA 1999;282: 1123-5.

11. Schwalbe RS, Stapleton JT, Gilligan PH. Emergence of vancomycin resistance in coagulase-negative staphylococci. N Engl J Med 1987;316:927-31.

12. Hiramatsu K, Hanaki H, Ino T, Yabuta K, Oguri T, Tenover FC. Methicillin-resistant Staphylococcus aureus clinical strain with reduced vancomycin susceptibility. J Antimicrob Chemother 1997;40:135-6.

13. Centers for Disease Control and Prevention. Staphylococcus aureus resistant to vancomycin-United States 2002. MMWR Morb Mortal Wkly Rep 2002;51:565-7.

14. Clinical Laboratory Standards Institute. Methods for dilution antimicrobial susceptibility tests for bacteria that grow aerobically. Approved Standard. Sixth Edition. CLSI document M7-A6. 2003.

15. Comité de l'antibiogramme de la societé francaise de microbiologie. Communiqué. Pathol Biol 1998;46:116.

16. British Society of Antimicrobial Chemotherapy. Revised guidelines for the control of MRSA infections in hospitals. J Hosp Infect 1998;39:253-90.

17. Olsson-Liljequist $\mathbf{B}$, Larsson $\mathbf{P}$, Walder $\mathbf{M}$, Miorner H. Antimicrobial susceptibility testing in Sweden. Scand $J$ Infect Dis 1997;105:13-23.

18. Ploy MC, Grélaud C, Martin C, de Lumley L, Denis F. First clinical isolate of vancomycin-intermediate Staphylococcus aureus in a French hospital. Lancet 1998;351:1212.

19. Bierbaum G, Fuchs K, Lenz W, Szekat C, Sahl HG. Presence of Staphylococcus aureus with reduced susceptibility to vancomycin in Germany. Eur J Clin Microbiol Infect Dis 1999;18:691-6.

20. Centers for Disease Control and Prevention. Staphylococcus aureus with reduced susceptibility to vancomycin-United States, 1997. MMWR Morb Mortal Wkly Rep 1997;46:765-6.

21. Smith TL, Pearson ML, Wilcox KR, Cruz C, Lancaster MV, Robinson-Dunn B et al. Emergence of vancomycin resistance in Staphylococcus aureus. Glycopeptide-Intermediate Staphylococcus aureus Working Group. N Engl J Med 1999;340:493-501.

22. Centers for Disease Control and Prevention. Staphylococcus aureus with reduced susceptibility to vancomycin-Illinois 1999. MMWR Mortal Wkly Rep 2000;48:1165-7.

23. Rotun SS, McMath V, Schoonmaker DJ, Maupin PS, Tenover FC, Hill BC et al. Staphylococcus aureus with reduced susceptibility to vancomycin isolated from a patient with fatal bacteremia. Emerging Infect Dis 1999;5:147-9.

24. Kim MN, Pai CH, Woo JH, Ryu JS, Hiramatsu K. Vancomycin intermediate Staphylococcus aureus in Korea. J Clin Microbiol 2000;38:3879-81.

25. Oliveira GA, Dell'Aquila AM, Masiero RL, Levy CE, Gomes MS, Cui L et al. Isolation in Brazil of nosocomial Staphylococcus aureus with reduced susceptibility to vancomycin. Infect Control Hosp Epidemiol $2001 ; 22: 443-8$

26. Arias CA, Reyes J, Zuñiga M, Cortés L, Cruz C, Rico CL et al. Multicentre surveillance of antimicrobial resistance in enterococci and staphylococci from Colombian hospitals, 2001-2002. J Antimicrob Chemother 2003;51:59-68.

27. Tenover FC, Lancaster MV, Hill BC, Steward CD, Stocker SA, Hancock GA et al. Characterization of staphylococci with reduced susceptibility to vancomycin and other glycopeptides. J Clin Microbiol 1998;36:1020-7.

28. Liñares J. The VISA/GISA problem: therapeutic implications. Clin Microbiol Infect 2001;7:8-15.

29. Avison MB, Bennett PM, Howe RA, Walsh TR. Preliminary analysis of the genetic basis for vancomycin resistance in Staphylococcus aureus strain Mu50. J Antimicrob Chemother 2002;49:255-60.

30. Navarre WW, Schneewind $\mathbf{O}$. Surface proteins of Gram-positive bacteria and their targeting to the cell wall envelope. Microbiol Mol Biol Rev 1999;63:174-229.

31. Hiramatsu K. Vancomycin resistance in staphylococci. Drug Resist Updat 1998;1:135-50.

32. Watanakunakorn C. Mode of action an in vitro activity of vancomycin. J Antimicrob Chemother 1984;14:7-18.

33. Hanaki H, Kuwahara-Arai K, Boyle-Vavra S, Daum RS, Labischinski H, Hiramatsu K. Activated cell-wall synthesis is associated with vancomycin resistance in methicillin-resistant Staphylococcus aureus clinical strains Mu3 and Mu50. J Antimicrob Chemother 1998;42:199-209.

34. Hanaki $\mathbf{H}$, Labischinski, Inaba $\mathbf{Y}$, Kondo $\mathbf{N}$, Murakami H, Hiramatsu K. Increase in glutamine nonamidated muropeptides in the peptidoglycan of vancomycin-resistant $S$. aureus strain Mu50. J Antimicrob Chemother 1998;42:315-20. 
35. Cui L, Murakami H, Kuwahara-Aria K, Hanaki H, Hiramatsu K. Contribution of a thickened cell wall and its glutamine non-amidated component to the vancomycin resistance expressed by Staphylococcus aureus Mu50. Antimicrob Agents Chemother 2000;44:2276-85.

36. Cui L, Ma X, Sato K, Okuma K, Tenover FC, Mamizuka EM, Gemmel CG et al. Cell wall thickening is a common feature of vancomycin resistance in Staphylococcus aureus. J Clin Microbiol 2003;41:5-14.

37. Jiang P, Peliska JA, Ninfa AJ. The regulation of Escherichia coli glutamine synthetase revisited: Role of 2-ketoglutarate in the regulation of glutamine synthetase adenylylation state. Biochemistry 1998;37:12802-10.

38. Bruckner R, Bassias J. Carbohydrate catabolism pathways and regulation in staphylococci. In: Fishetti VA, Novick RP, Ferretti JJ, Portnoy DA, Rood JI, editors. Gram Positive Pathogens. Washington DC:ASM Press; 2000. p.339-44.

39. Linnett PE, Strominger JL. Amidation and crosslinking of the enzimatically synthesized peptidoglycan of Bacillus stearothermophilus. J Biol Chem 1974;249:2489-96.

40. Sieradzki K, Tomasz A. A highly vancomycin-resistant laboratory mutant of Staphylococcus aureus. FEMS Microbiol Lett 1996;142:161-6.

41. Sieradzki K, Tomasz A. Inhibition of cell wall turnover and autolysis by vancomycin in a highly vancomycinresistant mutant of Staphylococcus aureus. J Bacteriol 1997;179:2557-66.

42. Wyke AW, Ward JB Hayes MV, Curtis NA. A role in vivo for penicillin-binding protein-4 of Staphylococcus aureus. Eur J Biochem 1981;119:389-93.

43. Finan JE, Archer GL, Pucci MJ, Climo MW. Role of penicillin-binding protein-4 in expression of vancomycin resistance among clinical isolates of oxacillin-resistant S. aureus. Antimicrob Agents Chemother 2001; 45:3070-5.

44. Boyle-Vavra S, Labischinski H, Ebert CC, Ehlert K, Daum RS. A spectrum of changes occurs in peptidoglycan composition of glycopeptide-intermediate clinical S. aureus isolates. Antimicrob Agents Chemother 2001;45:280-7.

45. Kuroda M, Kuwahara-Arai K, Hiramatsu K. Identification of the up- and down-regulated genes in vancomycin-resistant Staphylococcus aureus strains Mu3 and Mu50 by cDNA differential hybridization method. Biochem Biophys Res Commun 2000;269:485-90.

46. Kuroda M, Ohta T, Uchiyama I, Baba T, Yuzawa H, Kobayashi I et al. Whole genome sequencing of methicillin-resistant Staphylococcus aureus. Lancet 2001;357:1225-40.
47. Du W, Brown JR, Sylvester DR, Huang J, Chalker AF, So CY et al. Two active forms of UDP-Nacetylglucosamine enolpyruvyl transferase in Gram positive bacteria. J Bacteriol 2000;182:4146-52.

48. Komatsuzawa $\mathbf{H}$, Ohta $\mathrm{K}$, Sugai $\mathbf{M}$, Fujiwara $\mathbf{T}$, Glanzmann P, Berger-Bachi B et al. Tn551-mediated insertional inactivation of the fmtB gene encoding a cell-wall associated protein abolishes methicillin resistance in Staphylococcus aureus. J Antimicrob Chemother 2000;45:421-31.

49. Hsieh P. Molecular mechanisms of DNA mismatch repair. Mutat Res 2001;486:71-87.

50. Chambers HF. Methicillin resistance in Staphylococci. Molecular and biochemical basis and clinical implications. Clin Microb Rev 1997;10:781-91.

51. Muramaki K, Tomasz A. Involvement of multiple genetic determinants in high-level methicillin resistance in Staphylococcus aureus. J Bacteriol 1989;171:874-9.

52. Tanaka T, Okuzumi K, Iwamoto A, Hiramatsu K. A retrospective study on methicillin-resistant Staphylococcus aureus clinical strains in Tokyo University Hospital. J Infect Chemother 1995;1:40-9.

53. Liu C, Chambers H. Staphylococcus aureus with heterogeneous resistance to vancomycin: epidemiology, clinical significance and critical assessment of diagnostic methods. Antimicrob Agents Chemother 2003;47:3040-5

54. Hiramatsu K, Aritaka N, Hanaki H, Kawasaki S, Hosoda Y, Hori S et al. Dissemination in japanese hospitals of strains of Staphylococcus aureus heterogeneously resistant to vancomycin. Lancet 1997;350:1670-3.

55. Walsh TR, Bolmström A, Qwarnström P, Ho $\mathbf{P}$, Wootton M, Howe RA et al. Evaluation of current methods for detection of staphylococci with reduced susceptibility to glycopeptides. J Clin Microbiol 2001;39:2439-44.

56. Ariza J, Pujol M, Cabo J, Pena C, Fernández N, Linares $\mathbf{J}$ et al. Vancomycin in surgical infections due to methicillin-resistant Staphylococcus aureus with heterogeneous resistance to vancomycin. Lancet 1999;353:1587-8.

57. Schwaber MJ, Wright SB, Carmeli Y, Venkataraman L, DeGirolami PC, Gramatikova A et al. Clinical implications of varying degrees of vancomycin susceptibility in methicillin-resistant Staphylococcus aureus bacteremia. Emerging Infect Dis 2003;9:657-64.

58. Hiramatsu K. Vancomycin-resistant Staphylococcus aureus: a new model of antibiotic resistance. Lancet Infect Dis 2001;1:147-55.

59. Noble WC, Virani Z, Cree RG. Co-transfer of vancomycin and other resistance genes from Enterococcus faecalis NCTC 12201 to Staphylococcus aureus. FEMS Microbiol Lett 1992;72:195-8. 
60. Ray AJ, Pultz NJ, Bhalla A, Aron DC, Donskey CJ. Coexistence of vancomycin-resistant enterococci and Staphylococcus aureus in the intestinal tracts of hospitalized patients. Clin Infect Dis 2003;37:875-81.

61. Chang S, Sievert DM, Hageman JC, Boulton ML, Tenover FC, Downes PH. Infection with vancomycinresistant Staphylococcus aureus containing the vanA resistance gene. N Engl J Med 2003;348:1342-7.

62. Centers for Disease Control and Prevention. Vancomycin-resistant Staphylococcus aureusPennsylvania, 2002. MMWK Morb Mortal Wkly Rep 2002;51:902-3.

63. Whitener CJ, Park SY, Browne FA, Parent LJ, Julian K, Bozdogan B et al. Vancomycin-resistant Staphylococcus aureus in the absence of vancomycin exposure. Clin Infect Dis 2004;38:1049-55.

64. Centers for Disease Control and Prevention. Vancomycin-resistant Staphylococcus aureus-New York, 2004. MMWK Morb Mortal Wkly Rep 2004;53:322.
65. Walsh CT. Vancomycin resistance: decoding the molecular logic. Science 1993;261:308-9.

66. Weigel LM, Clewell DB, Gill SR, Clark NC, McDougal LK, Flannagan SE et al. Genetic analysis of a high level vancomycin-resistant isolate of Staphylococcus aureus. Science 2003;302:1569-71.

67. Tenover FC, Weigel LM, Appelbaum PC, McDougal LK, Chaitram J, McAllister S et al. Vancomycinresistant Staphylococcus aureus isolate from a patient in Pennsylvania. Antimicrob Agents Chemother 2004;48:275-80.

68. Anderson DI, Levin BR. The biological cost of antibiotic resistance. Curr Opin Microbiol 1999;2:489-93.

69. Tabaqchali S. Vancomycin-resistant Staphylococcus aureus: apocalypse now? Lancet 1997;350:1644-5.

70. Bush K. Vancomycin-resistant Staphylococcus aureus in the clinic: not quite Armageddon. Clin Infect Dis 2004;38:1056-7. 